\title{
Multi-wave Monitoring of the Gravitational Lensed Quasar Q0957+561
}

\author{
Vyacheslav N. Shalyapin ${ }^{1,2}$, Luis J. Goicoechea ${ }^{1}$ and \\ Rodrigo Gil-Merino ${ }^{1}$ \\ ${ }^{1}$ Universidad de Cantabria, 39005 Santander, Spain \\ email: vshal@ire.kharkov.ua \\ ${ }^{2}$ Institute for Radiophysics and Electronics, 61085 Kharkov, Ukraine
}

\begin{abstract}
We present X-ray (Chandra), ultraviolet (Swift/UVOT; $U$ band) and opticalinfrared (Liverpool Telescope; griz bands) continuum light curves of Q0957+561 observed in the first half of 2010. A cross-correlation analysis of the light curves shows that the $U$-band fluctuation leads the other variations at higher and lower energies. The study constrains the geometry of the continuum emission regions in a distant radio-loud AGN for the first time. We note that our work opens a new window in echo-mapping of high- $z$ AGNs with the use of lensed quasars, since the variability of some of the images of a given multiply-imaged quasar can be predicted in advance, provided there is a modest optical follow-up of the system.
\end{abstract}

Keywords. Gravitational lensing: strong, black hole physics, quasars: individual (Q0957+561).

\section{Motivation}

Optical monitoring of the double imaged quasar Q0957+561 $(z=1.41)$ enabled us to detect a very prominent increase in the brightness of the leading 'A' image between late 2008 and the middle of 2009 . From the observed delay of $\sim 14$ months between the two images, we predicted successfully that a repetition of the event would occur in the trailing 'B' image in the first half of 2010 (Goicoechea \& Shalyapin 2009).

\section{Observations}

A multi-wavelength monitoring campaign was organized in the first half of 2010 as a ground-based and space-based project, and involved X-ray, NUV, optical and NIR facilities. We obtained 6 light curves covering a large part of the electromagnetic spectrum:

- X-ray, 2-10 KeV Chandra/ACIS, 12 exposures, each for 3000 secs;

- U-band, Swift/UVOT, 35 exposures for 155-1092 secs;

- griz-bands, Liverpool Telescope: 55, 58, 50 and 45 exposures respectively per band, each for 120 secs.

The prominent flash of the 'A' image (2008/2009) is indeed repeated in the 'B' image in 2010. This fact demonstrates the intrinsic origin of the variability. The amplitudes of the variations significantly exceed their photometric errors. Moreover, the amplitude of the variability increases toward shorter wavelengths (higher energies).

In addition, we also detect, by chance, a clear U-shaped variation in image 'A'. It has a signal-to-noise ratio that is lower than the very prominent fluctuations in ' $\mathrm{B}$ ' (Fig. 1). 

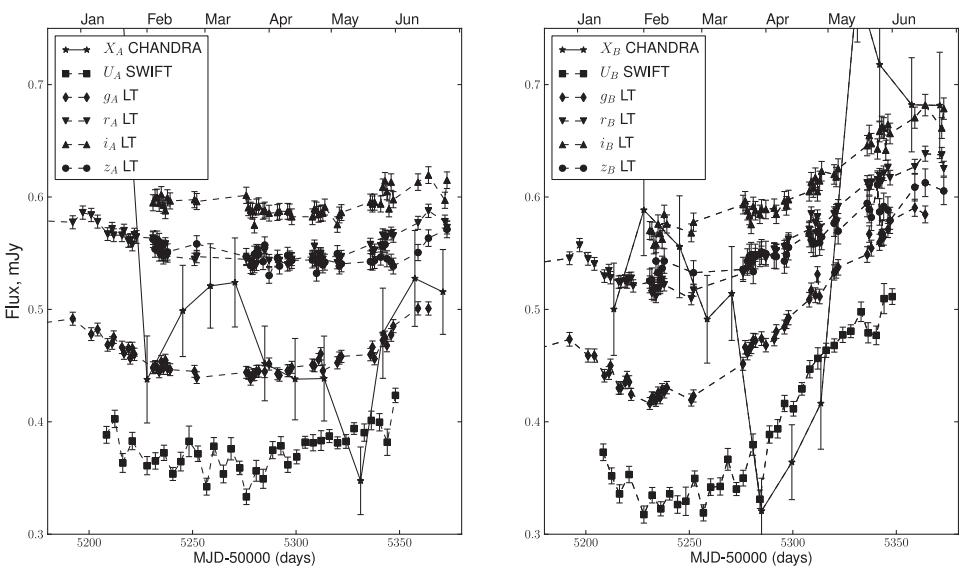

Figure 1. Variability in the 'A' (left) and 'B' (right) images of Q0957+561 in 2010.

\section{NUV/Optical/NIR Inter-Band Time Delays}

From the large variability of the ' $\mathrm{B}$ ' image in all bands we can estimate accurate interband time delays. The variability of the 'A' image can also be used to check our estimates from the ' $\mathrm{B}$ ' image data. We use both the $D^{2}$ and $\chi^{2}$ methods (Shalyapin et al. 2008 and references therein) to estimate time delays with respect to the $g$-band record $(1944 \AA$ in the source rest-frame). The inter-band delays from ' $A$ ' agree with the delays from ' $B$ '. As expected, the delays based on ' $A$ ' data have larger errors. The deviations between the two delay curves from ' $\mathrm{A}$ ' and ' $\mathrm{B}$ ' deserve more attention. The NUV fluctuations in 'A' and ' $\mathrm{B}$ ' lead the variations at other wavelengths. Our measurements suggest the presence of a central driving source that produces delays $\tau(\lambda) \sim \lambda^{4 / 3}$ for standard accretion disk rings around the central supermassive black hole (Collier et al. 1999). The driving source would emit at $\lambda<1438 \AA$ (source rest-frame); its flares would be reprocessed in the disk to trigger the observed NUV/optical/NIR variations.

\section{Origin of X-Ray Emission}

There is a $\sim 32$-day delay between the X-ray radiation and the $U$-band photons, so we can reject a standard corona-disk radiative coupling in which the disk variability is driven by a corona just above the black hole (Kazanas \& Nayakshin 2001). That result is also supported by X-ray reprocessing simulations and by the absence of X-ray reflection features in the spectrum of $0957+561$. The X-ray emitting region seems to be at the base of the Q0957+561 jet, at a height of $\sim 200 R_{g}$, or $0.05 \mathrm{pc}$ above the accretion disk. A central EUV source would drive the variability of Q0957+561 (Gil-Meruno et al. 2011).

\section{References}

Collier, S., Horne, K., Wanders, I., \& Peterson, B. M. 1999, MNRAS, 302, L24

Gil-Merino, R., Goicoechea, L. J., Shalyapin, V. N., \& Braga, V. F. 2011, ApJ, in press (arXiv:1109.3330)

Goicoechea, L. J. \& Shalyapin, V. N. 2009, The Astronomer's Telegram, no. 2228

Kazanas, D. \& Nayakshin, S. 2001, ApJ, 550, 655

Shalyapin, V. N., Goicoechea, L. J., Koptelova, E., Ullán, A., \& Gil-Merino, R. 2008, A\&A, 492, 401 